\title{
Familial Exudative Vitreoretinopathy (FEVR)
}

\section{Z Sardiwalla, H Ebrahim and VN Sukati}

Discipline of Optometry, School of Physiotherapy, Sport Science and Optometry, University of KwaZulu-Natal, Westville Campus, Private Bag X54001, Durban, 4000 South Africa

Received 28 January 2012; revised version accepted 28 May 2012

<z.sardiwalla@gmail.com><hanifazebrahim@gmail.com><mavegy@gmail.com>

\section{Introduction}

Familial Exudative Vitreoretinopathy (FEVR) is a progressive genetic disorder which presents with fundus features that are remarkably similar to retinopathy of prematurity (ROP) ${ }^{1}$. Patients, however, have no history of perinatal complications such as prematurity, incubation, supplemental oxygen or low birth weight ${ }^{2}$. This condition is also known as Criswick- Schepens Syndrome and characteristic features include failure of vascularisation of the temporal retinal periphery and retinal traction (as presented in this patient). Vitreoretinal attachments are also a common finding that may result in a ridge formation as well as vascular straightening and temporal dragging of the macula and optic disc ${ }^{3}$. Familial refers to the hereditary nature of the condition which in most cases is autosomal dominant or rarely $\mathrm{X}$ linked recessive ${ }^{3}$. Exudative describes the mass of lipid collections on the retina from leakage of blood vessels. The condition is bilateral but often asymmetrical in older patients ${ }^{4}$. Progressive myopia and anisometropia may also present in some patients ${ }^{1}$. Visual complications often arise when associated with cataracts, retinal detachment, glaucoma, vitreous haemorrhages, macula holes and giant retinal tears ${ }^{4}$.

Due to the condition often having an initially asymptomatic course but gradually presenting with a diversity of signs, it is often incorrectly diagnosed or even unrecognized ${ }^{1}$. Infants who are severely affected may be born blind whilst others with milder forms of the disease may be asymptomatic and only be diagnosed through fluorescein angiography ${ }^{5}$. The disease has been reported to show varied expressions even within family members ${ }^{6}$. The condition is very rare although this may be underestimated due to its silent nature in many patients?

Most previously reported cases on FEVR have highlighted some of the pathological signs commonly seen ${ }^{1,4}$. A selected few reports have elaborated on the binocular vision manifestations and assessment of BV related problems ${ }^{8,9}$. However, low vision assessment and management have not been highlighted in previous case reports. This is often the only appropriate management that an optometrist may provide. This clinical case therefore highlights how the optometrist can diagnose and manage patients who present with FEVR in practice.

\section{Case History}

A 19 year old Indian female student presented to the binocular vision clinic at the University of KwaZulu-Natal on the 17/08/2011 with complaints of decreased vision at distance even when using her current pair of single vision spectacles which were only four months old. She said she had been using spectacles for the last 10 years and that the vision in her left eye was worse than the right eye. She reported occasionally bumping into objects that were in her path suggesting visual field restriction. She sits in front in the lecture theatre in order to see the projector screen and uses large print notes to see more easily when studying. The patient reported positive to mild itching on the eyelid margin and longstanding severe tearing of unknown cause. A central or peripheral black dot 
or floater in the right eye was sometimes seen and mostly lasted for only a few minutes. This symptom started a few months ago but was not increasing in frequency. Observational assessment showed a mild ptosis (OS). The patient also had an alternating outward eye turn. Further probing elicited that the eye turn was present since birth and always on the left eye. She could not, however, identify whether the eye turned inward or outward but could tell that the squint was not getting any worse. The patient reported being occasionally bothered by the cosmetic aspect of the strabismus. At the age of 10 years, the patient re- ported that she had undergone binocular vision therapy and patching but could not remember the details of the therapy. The patient's prenatal, perinatal and postnatal history was unremarkable and she reported that she was not taking any medication or having any allergies. The patient reported no systemic illnesses however, she did report being diagnosed with FEVR 10 years ago by an ophthalmologist. She underwent retinal detachment surgery twice in 2007 (OS) and 2010 (OD). Her last ophthalmology visit was two months back and no new significant problems were noted. Her family ocular history revealed that her sis-
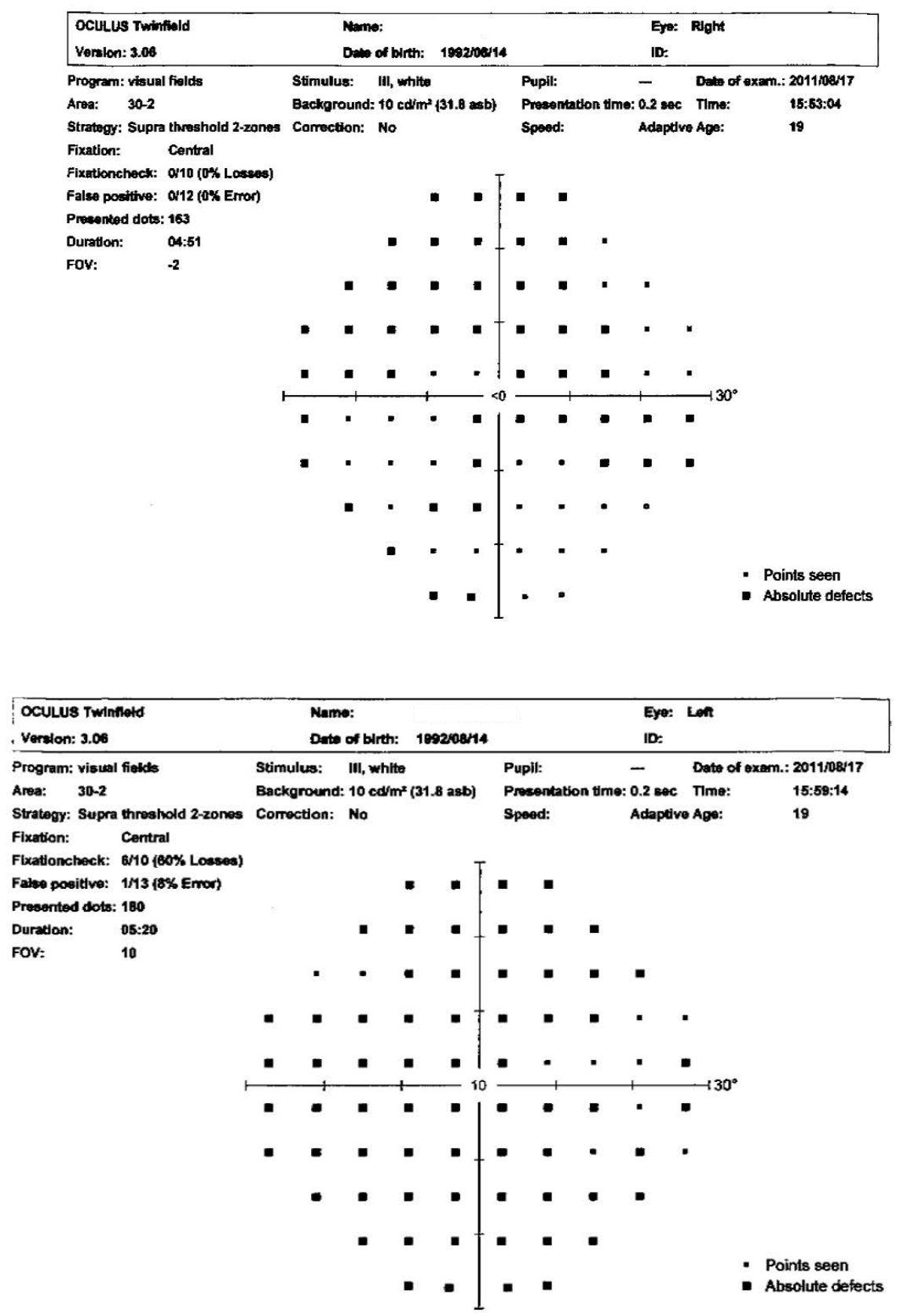

Figure 1: showing the results of the visual field testing using the Oculus Centerfield. Markedly restricted visual fields in both eyes. ter was also diagnosed with FEVR and her brother with keratoconus. Neither parent has been diagnosed with familial exudative vitreoretinopathy, however the father is hypertensive and wears spectacles. There was no history of blindness or strabismus in the family.

\section{Clinical Assessment and Findings}

Her unaided visual acuity with the LVRC $\log$ MAR Chart was $6 / 60$ for the right eye at distance and $1.6 \mathrm{M}$ at near with the left eye having a VA of 6/120 at distance and $4 \mathrm{M}+1$ at near. Aided visual acuity with the current pair of spectacles was $6 / 24$ at distance and $1.25 \mathrm{M}-3$ at near (OD), and $6 / 60+1$ at distance and $2.5 \mathrm{M}-1$ at near (OS). Binocularly, the patient had a VA of 6/24 at distance and $1.25 \mathrm{M}$ at near. The best corrected distance visual acuity OD was $6 / 30+2$ and OS $6 / 38-1$, with a manifest refraction of $-1.00 /-2.50$ $\mathrm{x} 130$ (OD) and -4.00 (OS), with no improvement in visual acuity using a pinhole. Amsler grid testing showed an absolute scotoma at the superior temporal region and a relative scotoma in the temporal region (OD). The left eye, however, revealed only an absolute scotoma that was documented in the temporal region. Visual field testing using 
both the tangent screen and the Oculus Centerfield revealed extensively restricted fields OU as shown in Figure 1.

Colour vision testing with the Farnsworth Munsell Panel D15 was normal (OU). Pupils were equally round and reactive to light with no afferent pupil defects noted (OU). Extraocular muscle movements were smooth and unrestricted. Intraocular pressure measured $9 \mathrm{mmHg}(\mathrm{OD})$ and $10 \mathrm{mmHg}$ (OS) with non-contact tonometry (Nidek NT530P). A quiet bulbar and palpebral conjunctiva with grade 1 blepharitis was noted (OU) on slit lamp examination. Evaluation of the tear film revealed a tear break up time of 4 seconds OD and 5 seconds OS. Ophthalmoscopy showed optically clear media (cornea, lens and vitreous). Evaluation of the posterior segment revealed sub retinal exudates in the inferior mid-periphery of the retina (OD). The optic disc and macula of both eyes appeared to be dragged temporally (Figure 2B and 2D) which is a common sign in this condition ${ }^{8}$. Vasculature in the temporal retina was absent with straightening of the vessels noted (OU) (Figure 2B). Scattered exudates were also noted below macula at the 6 o' clock position (OD) (Figure 2C). The macula area was not easily visible and absence of the foveal reflex was noted in both eyes.

\section{Binocular Vision Assessment}

Cover test without spectacles demonstrated a concomitant left esotropia (12 pd BO) and left hypotropia (25 pd BU) at distance. The deviation reduced to lateral orthotropia at near with a vertical deviation of $16 \mathrm{pd}$ BU (OS). There was no change in the magnitude of the strabismus with spectacles on. Angle kappa demonstrated $+2 \mathrm{~mm}$ (OD) and $+1.50 \mathrm{~mm}$ (OS). Hirschberg findings revealed a $+2 \mathrm{~mm}$ lateral deviation (OD) and a $+2 \mathrm{~mm}$ lateral deviation with 1 $\mathrm{mm}$ upward vertical deviation (OS). It could thus be seen that although observational assessment revealed an alternating XOT, a left SOT was actually present. This may be explained by the temporal dragging of the macula (macula ectopia) that forces the patient to fixate with the eyes in an abducted, and sometimes elevated position, which simulates the appearance of exotropia ${ }^{8}$.

Tests done to evaluate the presence of eccentric fixation using visuoscopy and the macular integrity tester trainer (MITT) were inconclusive. Normal retinal correspondence was found on the synoptophore with a subjective vertical angle of $11 \mathrm{pd}$ BD. Worth 4 dot testing revealed deep global suppression (OS) at distance and near. The filter bar also demonstrated
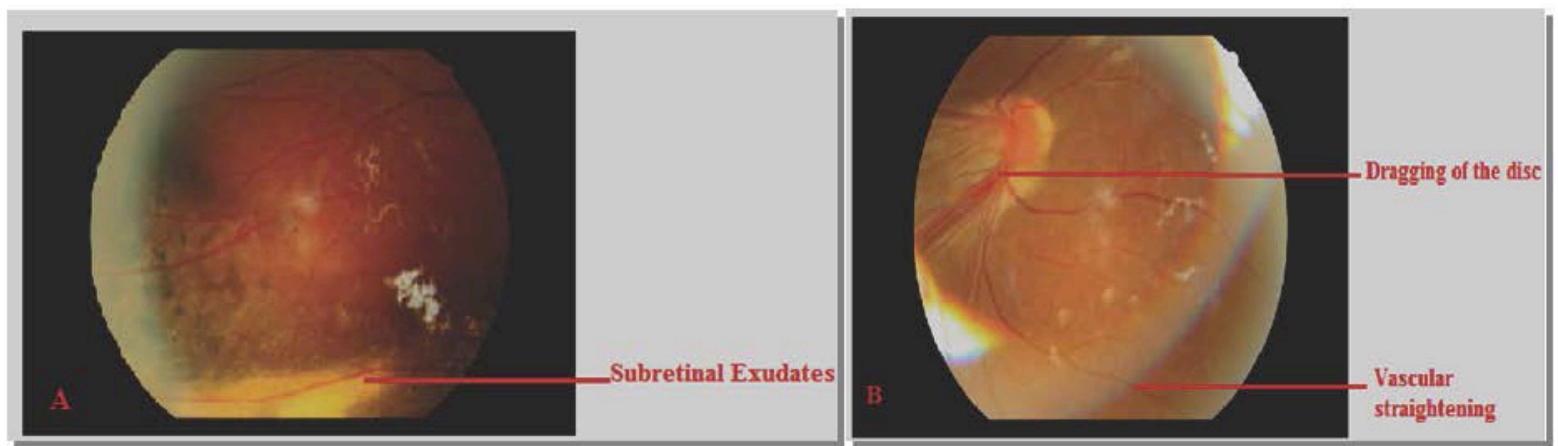

Figure 2A: Showing fundus photography findings of the right eye. A mass of subretinal exudates have settled inferiorly. Figure 2B shows temporal dragging of the optic disc and peripheral vascular straightening of the blood vessels in the right eye.
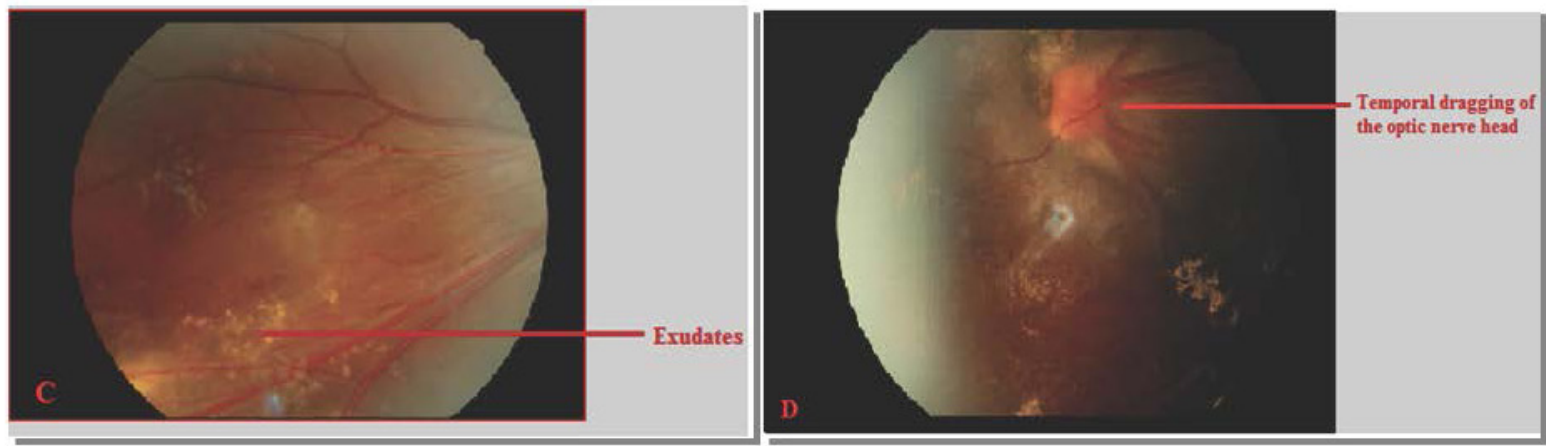

Figure 2C: showing exudates beneath the macula area and Figure 2D shows dragging of the optic nerve head in the left eye. 
deep suppression OS ( $>\# 17)$. Bagolini striated lenses and the synoptophore confirmed the suppression. On the synoptophore, intermittent suppression with Grade 1 and 2 targets was noted.

\section{Accommodative Status}

The monocular amplitudes of accommodation (OD 7.9 D and OS 7.5 D) and binocular (8.2 D) amplitudes were below the minimum of $10.25 \mathrm{D}$ for her age.

\section{Differential Diagnoses}

Other differential diagnoses that may be considered in a case of this type include the following:

a) Retinopathy of Prematurity: This accompanies a history of premature birth, perinatal complications, use of supplemental oxygen and majority of the time will develop vascularisation of the retina after involution of the disease ${ }^{4}$.

b) Eale's Disease: the vessels in this condition show inflammatory sheathing but no exudation or macular ectopia is seen ${ }^{4}$.

c) Coat's Disease: Often presents with masses of lipid exudates. This can be differentiated from FEVR by its unilateral presentation, male predilection and typical clinical features of light bulb shaped aneurysms ${ }^{10}$.

d) Norrie's Disease: This can mirror the advanced stages of FEVR but would also present with corneal opacification and micropthalmia with associated mental impairment and deafness ${ }^{10}$.

e) Toxocariasis: Toxocara granuloma present in children is accompanied with vitritis and uveitis ${ }^{4}$.

f) The above mentioned signs found in the other conditions may mimic FEVR were absent in this patient. Pathological findings confirmed stage III FEVR based on Pentergast's classification, which is similar to the classification of ROP, as shown in the table below ${ }^{1}$.

The patient was anisometropic with low myopia and moderate oblique astigmatism (OD) and moderate myopia (OS). A constant left small angle SOT was found at distance with a moderate-large left hypotropia at distance and near was found. This was of the divergence insufficiency type with deep suppression of the left eye. The patient had normal retinal correspondence with eccentric fixation tests being inconclusive.

\section{Management}

Due to the genetic nature of the condition, the options for managing patients with FEVR rest between intervention and monitoring 4 . Ophthalmology monitoring includes FA whilst intervention includes prophylactic photocoagulation in cases of peripheral neovascularisation and exudative detachment ${ }^{9}$. Optometric management includes treatment of the presenting symptoms through rehabilitation (visual aids). The patient's current pair of spectacles achieved similar visual acuity to the prescription found during the examination. The patient was therefore advised to continue using the current pair of spectacles. Since binocular vision functional prognosis was poor, no strabismus therapy was initiated. Surgery cannot be attempted to correct the vertical and horizontal deviation for a cosmetic cure due to the retinal detachments. No vertical prism was prescribed since the patient was asymptomatic.

Due to the poor visual acuity and visual field restriction, the patient qualifies to be a class one low vision patient. Although magnifiers are contraindicato-

Table 1: Showing the different stages of FEVR. This patient showed all signs of the disease.

\begin{tabular}{|l|l|}
\hline Stage & Findings \\
\hline I & Presence of avascular zone in the temporal retinal periphery. \\
\hline II & Presence of avascular zone, vitreous bands and pulling or neovascularisation. \\
\hline III & Stage two plus retinal detachment excluding fovea. \\
\hline IV & Retinal detachment subtotal, involving fovea. \\
\hline V & Total retinal detachment. \\
\hline
\end{tabular}


ry for restricted visual fields, however the patient had enough visual fields to allow the use of magnifiers. Low vision devices that were prescribed included a distance focusable Galilean hand held telescope of $3 x$ magnification $(\mathrm{VA}=6 / 9)$ and a near $2 \mathrm{x}$ dome shaped magnifier $(0.5 \mathrm{M}$ at $30 \mathrm{~cm})$. The patient was comfortable and motivated by the change in visual clarity offered by the devices. The patient was educated on the pathology and prognosis of the condition. Regular appointments with the ophthalmologist were emphasized. Optive was recommended bid to relieve the complaint of tearing which was found to be as a result of dry eye.

\section{Prognosis}

Therapy to restore binocular function is contraindicated due to a number of unfavourable factors which include underlying pathology, poor visual acuity, constant SOT since birth, deep suppression of the left eye, the age of patient and presence of a large vertical deviation. The only favourable factor was NRC. Therefore, prognosis for functional cure with binocular vision therapy was poor $(0-20 \%)^{11}$.

The prognosis of visual outcome for this patient is difficult to ascertain since the variable manifestations and progression associated with FEVR provide varied results in the literature ${ }^{1,4,12}$. Since the patient has already had two retinal detachments which represents stage three of the disease, visual prognosis is guarded ${ }^{1}$.

\section{Conclusion}

This case demonstrates the importance of careful case history and clinical observation skills that are important in diagnosing a patient with FEVR. This bilateral but often asymmetric condition should be carefully monitored, especially in patients who are symptomatic due to the high risk of retinal detachment $^{3}$. Furthermore, due to its autosomal dominant genetic nature, patient education and family screening are important considerations. Since the prognosis may sometimes be poor, as in this case, and other systems such as binocular vision are affected, the clinician needs to appreciate that due to the non functional cause of the strabismus, therapy will probably not be successful. An important consideration for visual re- habilitation is the effective use of low vision aids to assist the patient with poor visual acuity and visual field loss. Advice on future anticipations and possibly lifestyle modifications should be discussed with the patient.

\section{References}

1. Yang C, Chen S, Yang M. Excessive myopia and anisometropia associated with Familial Exudative Vitreoretinopathy. Chang Gung Med J 2002256 388-392.

2. Kondo H, Hayashi H, Oshimi K, Tahiri T, Hayashi K. Frizzled 4 gene (FZD4) mutations in patients with familial exudative vitreoretinopathy with variable expressivity. $\mathrm{Br} J$ Ophthalmol 200387 1291-1295.

3. Kanski JJ. Clinical ophthalmology: A systematic approach. Sixth Edition. New York: Butterworth Heinemann, 2008 $\mathrm{p} 8$.

4. Shukla D, Singh J, Sudheer G, Soman M, John RK, Ramasamy K, Perumalsamy N. Familial exudative vitreoretinopathy (fevr). Clinical profile and management. Indian J Ophthalmol $200351323-328$.

5. Van Nouhuys CE. Signs, complication, and platelet aggregation in familial exudative vitreoretinopathy. Am J Ophthalmol 1991111 34-41.

6. Canny CL, Oliver GL. Fluorescein angiographic findings in familial exudative vitreoretinopathy. Arch Ophthalmol 197694 114-120.

7. Toomes C, Downey L. Familial exudative retinopathy, autosomal dominant 2011 Pubmed Bookshelf Available at http://www.ncbi.nlm.nih.gov/books/NBK1147/.

8. Rosenbaum AL, Santiago AP. Clinical strabismus management: principles and surgical techniques. Philadelphia: WB Saunders Company, 1999 p179.

9. Pendergast SD, Trese MT Familial exudative vitreoretinopathy: Results of surgical management. Ophthalmol 1998 105 1015-1023.

10. Feiner L, Prenner JL. Retinal vascular disease in Children: A look at the clinical and genetic features that make a difficult diagnosis easier Rev Ophth 2006 111-116.

11. Griffin J. Binocular anomalies: diagnosis and vision thera$p y$, Third Edition. New York: Butterworth and Heinemann, 2002 p 229.

12. Fulton AB, Hansen RM, Petersen RA Vanderveen DK. The rod photoreceptors in retinopathy of prematurity: an electroretinographic study. Arch Ophthal 2001119 499-505. 